\title{
590 ANTI-CD33 ACTINIUM-225 TARGETED RADIOIMMUNOTHERAPY ENHANCES THE BIOLOGIC ACTIVITY OF ANTI-CD47 ANTIBODY IMMUNOTHERAPY IN PRECLINICAL MODELS OF ACUTE MYELOID LEUKEMIA
}

Sagarika Pachhal, Emily Greer, Jesse Hwang, Qing Liang, Mary Chen, Eileen Geoghegan, Helen Kotanides, Dale Ludwig, Denis Beckford*. Actinium Pharmaceuticals, New York, NY, USA

Background Actimab-A, the anti-CD33 antibody lintuzumab armed with the radioisotope Actinium-225 (Ac-225), has demonstrated single agent antileukemic effects in patients with relapsed or refractory acute myeloid leukemia (AML). Up-regulation of CD47, a macrophage checkpoint that suppresses phagocytosis, is one mechanism by which myeloid malignancies such as AML can evade targeting by the innate immune response. Therapeutic blocking antibodies against this pathway have shown early clinical promise. We hypothesized that Actimab-A will enhance phagocytosis in AML cells by specifically upregulating calreticulin (CRT), a pro-phagocytic signal. Moreover, we hypothesized that combination of the anti-CD33 antibody radioconjugate (ARC) and CD47 blocking antibody could act in synergy to enhance therapeutic outcomes in AML compared to single agent. In this study, we examined, for the first time, the potential mechanistic benefit of combining the anti-CD33 ARC armed with Ac-225 or Lutetium-177 (Lu-177) and a CD47 blocking antibody, using in vitro and in vivo human AML preclinical models.

Methods Lintuzumab was conjugated with p-SCN-DOTA and radiolabeled with Ac-225 or Lu-177. The biological activity of both radioconjugates was examined using human recombinant CD33 and receptor positive AML cells. The cytotoxic effect of radioconjugates and the ability to upregulate CRT was evaluated using XTT assay and flow cytometry, respectively, in a panel of CD33 expressing cells. To assess the therapeutic combination of anti-CD33 ARC and CD47 antibody in vitro, a flow cytometry macrophage phagocytosis assay was used. We further evaluated the therapeutic efficacy in vivo of anti-CD33 ARC and CD47 antibody combination in human AML mouse model.

Results The anti-CD33 ARCs have similar binding properties to native antibody and demonstrate specific cell cytotoxicity. We show ARC-mediated upregulation of cell surface CRT in a panel of CD33 expressing AML cells. Furthermore, the in vitro combination of CD33 targeting ARC and CD47 blocking antibody enhances macrophage mediated phagocytosis of AML cells compared to each monotherapy. Interestingly, the in vivo anti-CD33 ARC and CD47 antibody combination demonstrates a significant increase in survival and reduces toxicity in a human AML preclinical model.

Conclusions Our findings suggest a novel synergistic mechanism whereby the CD33 ARC targeted radiation induces upregulation of CRT, thereby potentiating a pro-phagocytic innate immune response in combination with anti-CD47 blocking antibody. More importantly, clinical translation of this approach could enhance therapeutic efficacy in AML and warrants further preclinical exploration.

Ethics Approval All animal studies were approved by IACUC.

http://dx.doi.org/10.1136/jitc-2021-SITC2021.590 